\title{
ACTUACIÓN EFECTIVA DEL ÁREA DE SEGUIMIENTO DE ACUERDOS REPARATORIOS
}

\section{EFFECTIVE PERFORMANCE OF THE REPAIR AGREEMENTS MONITORING \\ AREA}

\author{
Gloria María Jerónimo-García ${ }^{1} *$ (D). \\ 1. Escuela Judicial del Estado de Tabasco, México.gabriela.gloria_pocajonta@hotmail.com \\ * Autor de correspondencia: Gloria María Jerónimo-García, correo electrónico: gloria_pocajonta@hotmail.com
}

\section{RESUMEN}

Este artículo describe y explica lo que acontece después de celebrar un procedimiento, que da como resultado un acuerdo reparatorio (AR), utilizando mecanismos alternos de solución de controversias (MASC), previsto en la Ley Nacional de Mecanismos Alternativos de Solución de Controversias en Materia Penal (LNMASCMP), dependiendo que en caso proceda; conciliación, mediación y la junta restaurativa, los cuales se celebran entre víctima u ofendido y el imputado. Una vez celebrado dicho AR, existe un área que se encarga del seguimiento, que tiene que cumplir con cierta funcionalidad. La reforma del 2008 a la Constitución Política de los Estados Unidos Mexicanos (CPEUM) en su artículo 17, cuarto párrafo, da la creación de la LNMASCMP quien, a partir de su publicación en 2014, rige los mecanismos alternativos en materia penal. Sin embargo, se carece del conocimiento sobre el manejo o gestión después de celebrar un AR, en particular del área de seguimientos de RP de la Fiscalía General del estado de Tabasco (FGE), así como la función que desempeña al tener conocimiento de la suscripción de AR diferido, misma que se encuentran estipuladas en el numeral 36 de la (LNMASC) en sus distintas fracciones.

Palabras claves: Acuerdo reparatorio; fiscalía; justicia alternativa; Ley Nacional de Mecanismos Alternativos de Solución de Controversias en Materia Penal.

Cómo citar:

Jerónimo-García, Gloria María. (2021). Actuación efectiva del área de seguimiento de acuerdos reparatorios. Revista de Investigaciones Universidad del Quindio, 33(S2), 174-182. https://doi. org/10.33975/riuq.vol33nS2.629 


\begin{abstract}
This article describes and explains what happens after holding a procedure, which results in a reparatory agreement (AR), using alternative dispute resolution mechanisms (MASC), provided for in the National Law of Alternative Mechanisms for the Resolution of Controversies in Criminal Matters (LNMASCMP), depending on the appropriate case; conciliation, mediation and the restorative meeting, which are held between the victim or offended and the accused. Once said AR has been held, there is an area that is in charge of monitoring, which has to comply with certain functionality. The 2008 reform to the Political Constitution of the United Mexican States (CPEUM) in its article 17, fourth paragraph, creates the LNMASCMP which, as of its publication in 2014, governs alternative mechanisms in criminal matters. However, there is a lack of knowledge about the handling or management after holding an RA, in particular of the PR monitoring area of the Attorney General's Office of the State of Tabasco (FGE), as well as the role it plays when having knowledge of the subscription deferred AR, which are stipulated in numeral 36 of the (LNMASC) in its different sections.
\end{abstract}

Keywords: Reparatory Agreement; prosecutor's office; alternative justice; National Law of Alternative Mechanisms for the Resolution of Disputes in Criminal Matters.

\title{
INTRODUCCIÓN
}

La importancia de analizar el área de mecanismos alternativos en específico de seguimiento de acuerdos, de la Fiscalía General del Estado de Tabasco, se deriva que los usuarios carecen de conocimiento sobre formalidades a seguir después de la redacción de un acuerdo reparatorio formal, ante el Órgano Especializado en Mecanismos Alternativos de Solución de Controversias en Materia Penal, por lo cual se abordará a groso modo el camino a seguir unas vez firmado un acuerdo reparatorio diferido o en su caso el incumplimiento del mismo, que se encuentran establecidos en el artículo $36^{\circ}$ de la Ley Nacional de Mecanismos Alternativos de Solución de Controversias en Materia Penal (LNMASCMP).

Este artículo se divide en tres apartados, de los cuales el primero de ellos abordar los casos exitosos en el uso de los métodos alterno, se describen las herramientas para el seguimiento de acuerdo, efectos del cumplimiento del acuerdo y los efectos del incumplimiento del acuerdo, por último, se aborda la efectividad del seguimiento de acuerdo reparatorio diferidos.

\section{METODOLOGÍA}

En la comprensión de los apartados de este artículo se empleó el método exegético que comprende la interpretación de diversas leyes, códigos, reglamentos, asimismo, la consulta de la doctrina a través de diversos autores expertos en el tema, lo que sirvió como apoyo para ampliar la noción del trámite que se continua una vez redactado un acuerdo reparatorio ante el Órgano. La integración del método que se utiliza para el desarrollo de este artículo es el método deductivo, que va de lo general a lo específico, con el que se pretende explicar la importancia de área de seguimiento de acuerdo del Órgano para materializar la figura jurídica de acuerdo reparatorio. 


\section{RESULTADOS}

\section{1.- Éxitos en el uso de los métodos alternos.}

Con los mecanismos alternos el Estado puede otorgar soluciones eficaces a los conflictos de distintas índoles, evitando el incremento de asuntos en los centros de administración de justicia, que en ocasiones se encuentran colapsados por la cantidad de asuntos.

En la reforma del de junio de 2008 a la Constitución Política de los Estados Unidos Mexicanos, anexaron los mecanismos alternativos de solución de controversias en su artículo 17, cuarto párrafo que actualmente a la fecha señala: "Las leyes preverán mecanismos alternativos de solución de controversias. En la materia penal regularán su aplicación, asegurarán la reparación del daño y establecerán los casos en los que se requerirá supervisión judicial". Por su parte la Ley Nacional de Mecanismos Alternativos, señala que a través de los mecanismos alternativos se propicie la solución de controversias. Podemos ver que dentro de La ley Orgánica de la Fiscalía General del Estado de Tabasco, señala que la fiscalía contara con órganos especializados en mecanismos alternativos de solución de controversias (Art. 17).

Es importante definir el término acuerdo reparatorio, el cual se refiere al acuerdo entre el imputado y la victima u ofendido, en que el primero repara de algún modo que resulte satisfactorio para la segunda las consecuencias dañosas del hecho que se persigue penalmente y que, una vez aprobados por el MINISTERIO Público o el juez de control y cumplidos en sus términos, tienen como efecto la conclusión del caso (Horvitz \& López, 2003).

Antes de redactar cada uno de los ejemplos de los procedimientos exitosos al uso de los mecanismos alternativos, vamos aclarar que los intervinientes en un procedimiento en donde debe existir un solicitante y un requerido, siendo el solicitante la persona que requiere el servicio del Órgano y por su parte el requerido, quien será la persona que invitaremos a participar en un procedimiento alterno.

Requerido: La persona física o moral convocada para solucionar la controversia penal mediante la aplicación de un mecanismo alternativo (LNMASCMP, art 3, fracción XI)

Solicitante: La persona física o moral que acude a los Órganos de Justicia Alternativa, con la finalidad de buscar la solución de una controversia penal (LNMASCMP, art 3, fracción XIII).

\subsection{Mediación (ejemplo)}

En caso que nos ocupa se trata del delito de lesiones, procedimiento de mediación celebrado entre el solicitante y el requerido (Yesenia y Karla), Yesenia se encontraba en el súper de compras, cuando sintió que a su espalda le jalaban el cabello y era agredida físicamente por la persona de Karla, quien a su vez le refería palabras altisonantes, luego Karla se retiró del lugar, inmediatamente posterior a lo ocurrido Yesenia denunció los hechos ante el Centro de Procuración de Justicia de Nacajuca Tabasco, cuando el Fiscal del Ministerio Público, tuvo conocimientos de los hechos de manera instantánea remitió dicha carpeta de investigación ante el Órgano Especializado en Mecanismos Alternativos de Solución de Controversia en Materia Penal, donde se inició un procedimiento de mediación, el cual se llevó efecto dentro de los términos legales, las partes pudieron llegar a un Acuerdo Reparatorio donde ambas participantes se comprometieron a no molestarse nuevamente y hacer efectiva una reparación 
económica con apego a la Norma Jurídica aplicable dentro de los 30 días siguientes a la suscripción del Acuerdo, dicho Acuerdo fue remitido al área de seguimiento de la Dirección del Órgano Especializado en Mecanismos Alternativos de Solución de Controversia en Materia Penal, con sede en la ciudad de Villahermosa, Tabasco, donde le dieron rastreo correspondiente y al llegar a la fecha estipulada para su cumplimiento se le informó al fiscal del Ministerio Público el cumplimiento del mismo, para su debido trámite, el cual dio como resultado Archivo de la Carpeta de Investigación.

\subsection{Conciliación (ejemplo)}

Ana y juan no se conocían, tuvieron un hecho de tránsito por la forma de conducir sus unidades, la causa probable hizo responsable a Juan, quien fue el requerido a solicitud de Ana, en la diligencia de carácter conciliatorio Juan se comprometió a realizar un pago por la Reparación de daños de $\$ 8,000$ pesos (ocho mil pesos 100/00 m.n.) a lo cual Ana aceptó que se realizará en 4 parciales cada una por la cantidad de $\$ 2,000$ pesos (dos mil pesos 100/00 m.n.) a cada fin de mes, sin embargo Juan tuvo un improvisto y se atrasó en la última fecha de pago y el área de seguimientos de acuerdo se percató de esa demora, los mando a requerir a las partes asignado fecha y hora para darle seguimiento al acuerdo celebrado con fechas anteriores donde asistió Juan y refirió sus motivos por el cual había tenido esa demora y solicitó en presencia de Ana una disculpa y en ese acto hizo el último pago, lo que trajo como consecuencia como el ejemplo anterior el archivo por cumplimento de acuerdo reparatorio de la Carpeta de Investigación (FGET, 2019).

\subsection{Junta restaurativa (ejemplo)}

Existía un conflicto entre dos hermanos por una cuestión de la hija de la solicitante u ofendida, el ofensor difamo a la hija de la hermana y eso causo molestia a la solicitante hasta llegara los empujones y uso de vocabulario inapropiado, en la junta restaurativas participaron la hija de la solicitante y la madre de ambos participantes debido a que resultó afectada al ver a sus hijos dentro de este conflicto ya que nunca había ocurrido, el requerido reconoció su culpa y muy consiente que su actuar no fue el apropiado, firmaron un acuerdo de perdón y su vínculo familiar se volvió a construir, de manera inmediata se le informo al Fiscal del Ministerio Público la celebración de un acuerdo Inmediato, quien notifico en la misma fecha el archivo de la carpeta de investigación por celebración y cumplimiento de acuerdo (FGET, 2019).

Estos ejemplos muestran los éxitos alcanzados a través del uso de los mecanismos alternos (conciliación, mediación y junta restaurativa) por medio del Órgano Especializado en Mecanismo Alternativos de Solución de Controversia en materia penal de la Fiscalía del Estado de Tabasco que al dar un seguimiento pronto y adecuado se culmina de maneras satisfactoria y esto hace que el trámite de la integración de un carpeta de investigación o dentro de un procedimiento penal en sede judicial, la víctima u ofendido reciba su reparación de daño de manera eficaz, para hace efectiva o un debido seguimiento de los acuerdos suscrito ante el Órgano se cuenta con herramientas que la Ley Nacional de Mecanismos Alternativos de Solución de Controversia de materia penal lo cual se describa en las líneas siguientes.

\section{2.- Herramientas para el seguimiento de acuerdo}

El Órgano cuenta con un área de seguimiento, la cual se encarga y tiene la obligación de monitorear e impulsar el cumplimiento del acuerdo alcanzado por los Intervinientes en el Mecanismo Alternativo (LNMASCMP:36, Pfo. $1^{\circ}$.) 
A razón de la creación del Área de seguimiento de Acuerdo la Fiscalía del Estado de Tabasco incluye dentro del Órgano y su Organigrama dicha función del mismo, así como el órgano cuenta con un manual donde se detalla, la función y procedimientos de la Dirección de Mecanismos Alternativos de Solución de Controversia en Materia Penal de la Fiscalía General del estado de Tabasco. (FGET, 2020).

La misma ley a demarcado la unidad administrativa la cual le denominó Órgano, quien realiza las tareas de aplicación de los MASC. La definición de Órgano conforme la Ley es la institución especializada en mecanismos alternativos de solución de controversias en materia penal perteneciente a la federación o entidades federativas (LNMASCMP, 2014).

Tal como lo estable la LNMASCMP en sus distintas fracciones del numeral 36, estos instrumentos podrán consistir: a) Apercibimiento a los Intervinientes para el caso de incumplimiento del Acuerdo; b). Visitas de verificación; c). Llamadas telefónicas; d). Recepción o entrega de documentos, pagos, bienes u objetos; e). Citación de los Intervinientes y demás personas que sean necesarias; f). Envío de correspondencia o comunicación, pudiendo usar medios electrónicos, y g). Cualquier otra medida necesaria para el cumplimiento del Acuerdo de conformidad con los principios y disposiciones establecidas en esta Ley.

Es de especial relevancia el seguimiento del cumplimiento de los acuerdos, pues el objetivo es concluir el asunto y el conflicto penal deje de estar permanente. Este tipo de acciones que el Área de seguimiento realiza hay una de tipo de fiscalización cuando se refiere a las visitas de verificación, llamadas telefónicas, recepción o entrega de documentos, pagos, bienes u objetos, citación de los intervinientes y demás personas que sean necesarias, envío de correspondencia o comunicación, pudiendo usar medios electrónicos, la segunda seria de naturaleza jurídica denomina apercibimiento, que sería la advertencia de que al no cumplir el acuerdo se seguirá la integración del procedimiento penal, en relación a lo dispuesto por el artículo 37 de la LNMASCMP.

Solo como forma de realizar una comparación entre el estado de puebla y tabasco, en la estructura del Órgano Especializado este se encuentra con diferente departamento encargado de realizar dicho seguimiento como son, con una unidad de atención temprana; con orientadores, invitadores, facilitadores, validadores; con un área de seguimientos (la cual tiene total importancia para monitorear la eficacia de la solución alterna); con alimentadores de la base de datos y con el personal administrativo según las capacidades presupuestarias de cada entidad (Basulto, 2019).

Para alcanzar una eficacia en el seguimiento ante el Órgano se mantiene una comunicación constante con las partes del acuerdo, para facilitar el cumplimiento de las obligaciones contraídas. Si percibe el área de la existencia del incumplimiento por partes de las personas obligadas, se le exhorta al cumplimiento del acuerdo o citar a reuniones de revisión en compañía del facilitador o mediador del órgano mismo que llevó el mecanismo. se tendrá que realizar reuniones de revisión donde se exprese el porqué del incumplimiento, para poder construir un resultado satisfactorio entre ambas partes, para alcanzar la reparación de daño. (LNMASCMP, Artículo 38).

Los acuerdos reparatorio se sustentan en los que tomen la víctima y el imputado, en los cuales el imputado repara a satisfacción el daño causado a la víctima. Una vez aprobado o cumplidos tales acuerdos, la consecuencia inmediata es la extinción de la acción penal o en su casa el incumpliendo del mismo la continuidad del proceso o investigación, la cual de describirá a continuación. 


\section{1- Efectos del cumplimiento del acuerdo}

Como lo señala el CNPP los acuerdos reparatorio una vez aprobados por el Ministerio Público o el Juez de control y una vez cumplidos en todos sus términos, tiene como consecuencia la extinción de la acción penal. (art. 186)

Continúa diciendo el CNPP en su numeral (art 190), los trámites de los acuerdos reparatorio dependiendo en la etapa en que se celebren tendrá que ser aprobados por el Juez de control a partir de la etapa de investigación complementaria o en su caso por el Ministerio Público en la etapa de investigación inicial.

Cuando el Juez le corresponde avalar los acuerdos, siempre y cuando el imputado cumpla con las obligaciones pactadas, deberá ordenar el archivo definitivo o sobreseimiento y declarar extinguida la acción penal. Estos efectos se producen con el cumplimiento de lo acordado, no cuando se produce el acuerdo (Vasconcelos, 2012).

En cualquiera de los casos una vez aprobado el cumplimiento del Acuerdo, se resolverá de inmediato sobre la extinción de la acción penal o el sobreseimiento del asunto según corresponda. La resolución emitida por el Juez tendrá efectos de sentencia ejecutoriada (LNMASCMP:35)

\section{2 efectos del incumplimiento del acuerdo}

Es muy importante que el acuerdo suscrito entre los intervienes en un proceso (solicitante y requerido) sean cumplidos, pero cuando esto no ocurre haya una respuesta del sistema. Por ellos si le requerido o imputado depende de la etapa de la suscripción del acuerdo no cumple con las obligaciones y compromisos pactados sin causa justa la víctima, ofendido o el Fiscal del Ministerio Público pueden informarlo al juez, al facilitador o en este caso al área de seguimiento, quienes deberán llevar a cabo una audiencia para constatarlo y si considera después de escuchar a ambas partes que hay incumplimiento injustificado o falta de interés del imputado a cumplirlo se deberá informar al juez o al fiscal del ministerio público dicho incumplimiento el cual dará a lugar la continuidad del procedimiento o carpeta de Investigación.

La LNMASCMP señala que una vez corroborado dicho incumplimiento deberá aplicar los apercibimientos, el Órgano deberá llevar acabo la reunión de revisión para revisar los motivos de incumplimiento y exhortarlos a la parte incumplidora y en la media de lo posible remedie la omisión (artículo 38). Es por ello, que si no se logra formar un esquema que garantice que ahora si se llevará un cumplimento, el área de seguimiento comunicara al facilitador, al ministerio público o al juez para que de continué con el proceso penal, si el imputado incumplió alguna de las clausulas o parte de lo pactado deberá el fiscal del ministerio público tomarlo en cuenta para la reparación de daño.

\section{3.- Efectividad del seguimiento de acuerdo reparatorio.}

La finalidad de los procesos alternativos es buscar que los participantes dialoguen acerca del conflicto (Silva, 2020) que presentan al momento de solicitar el procedimiento alternativo con ayuda de un facilitador acatando las reglas y los principios rectores de los mecanismos alternativos y con ayuda del facilitador, las partes puedan alcanzar un acuerdo, por alguno de los mecanismos (conciliación, mediación y la junta restaurativa). 
[Los mecanismos alternativos (conciliación, mediación y la junta restaurativa) de controversia en materia penal tiene como finalidad propiciar, a través del dialogo, la solución de las controversias que surjan entre miembros de la sociedad con motivo de la denuncia o querella referido a un hecho considerado como delito, mediante procedimientos basados en la oralidad, la economía procesal y la confidencialidad (LNMASCMP: 1, Pfo.2)].

Los mecanismos en el sistema de justicia penal, es una eficaz alternativa para la justicia adversarial, para su aplicación se hacen uso de estos principales ordenamientos como son: Tratados internacionales, CPEUM, LNMASCMO, CNPP, entre otros. Los cuales son utilizados para la correcta aplicación y desarrollo de los órganos especializados en mecanismos alternativos de las fiscalías de los Estados.

El órgano especializado dependerá de las Fiscalía General de la República o de las Fiscalías General de los Estados, los cuales cada una de ellas deberán contar con un Órgano Especializado en mecanismos alternativos de resolución de controversias... (LNMASCMP: artículo 40), mismo que nos sigue diciendo que entre los fines de los órganos especializados, son, tramitar los Mecanismos Alternativos, realizar acciones tendientes al fomento de la cultura de la paz, estandarizar programas de capacitación continua para su personal, difundir y promover la utilización de los Mecanismos Alternativos y la conservación de la bases de datos. (Javier, 2016). Con independencias de las Fiscalías ya sea federal o estatales también tendrán que contar con Órganos Especializados.

Entre las atribuciones del órgano en específico del área de seguimiento, está obligado a conservar una base de datos de los procedimientos que ingresan al órgano, donde se registren el estatus en el que se encuentran y su resultado final, debiendo actualizar de manera constante la base y llevar estudios estadísticos del funcionamiento del servicio, donde se observara el porcentaje de acuerdos cumplidos e incumplidos y los casos de reingresos de los Intervinientes (LNMASCMP: 43, Pfo.10).

En relación a la base que maneja el Órgano, se cuenta con una base nacional con la información ya descrita anteriormente a la cual podrán acceder los Órganos; el requisito de este será a cargo por la Conferencia y el Consejo y administrada por el Centro Nacional de Información del Secretariado Ejecutivo del Sistema Nacional de Seguridad Pública. Los Poderes Judiciales están obligados a dar la información correspondiente a la fiscalía federal y de las entidades federativas (LNMASCMP: 43, Pfo.2o)

La forma de conocer un resultado positivo o exitoso de los procedimientos alternativos, cuando al finalizar el procedimiento alternativo, los intervinientes alcanzan un acuerdo y el mismo se cumple dentro de los términos estipulados o en su caso cuando se haga una revisión en los nuevos términos del acuerdo y estos mismos se cumplan. Una vez de tener conocimiento del cumplimiento o incumplimiento de los acuerdos el encargado del área de seguimiento de acuerdos del Órgano, informa al Facilitador, al Ministerio Público, al Juez competente, a efectos que determine las consecuencias jurídicas respectivas (LNMASCMP: 37).

Para el logro de una eficaz intervención del órgano, deber comunicarse periódicamente con los suscriptores de los Acuerdos si la naturaleza del caso lo permite, para verificar o facilitar el cumplimiento de las obligaciones contraídas.

Los ventajas de acudir ante el Órgano especializado hace que los protagonistas de los conflictos al hacer uso de estos mecanismos el tiempo es menor que llevar un procedimiento penal pues ellos 
mismos deciden los plazos y términos a diferencia del proceso que un tercero decide a quien le asiste la razón en un término mayor de treinta días, el costo es menor, se conoce que la impartición de justicia es gratuita sin embargo hay personas que deciden contratar expertos en la materia para hacer valer sus derechos, el cual genera un costo a diferencia de acudir ante el órgano quien cuenta con persona especialista en la materia y certificada quien brinda el servicio gratuito, al acudir a un procedimiento alterno son las partes que establecen sus ritmos y deciden si es pertinente o no las propuestas expuestas para la solución de sus conflictos o decidir continuar con el procedimiento penal donde las partes están siempre supeditadas a las decisiones que toma un tercero, hay un control de resultados pues este se basa en las propuestas de los participantes, el acuerdo alcanzado por el mecanismos es vinculante pues ahí se adquieren derechos y obligaciones que se contraen de manera voluntaria libremente adoptados durante el desarrollo del procedimiento.

El artículo 7 de la Asamblea General de la ONU resolución 40/34 del 29 de noviembre 1985, otorga a las partes acceso a los mecanismos que garanticen la reparación de daño el cual en su numeral 7, dice que: Se utilizarán, cuando proceda, mecanismos oficiosos para la solución de controversias, incluidos la mediación, el arbitraje y las prácticas de justicia consuetudinaria o autóctonas, a fin de facilitar la conciliación y la reparación en favor de las víctimas.

La ONU de manera oficiosa considera necesario que se agote dentro de un procedimiento una solución alterna al que los usuarios del sistema no están acostumbrados, claro es de tomarse en cuanta en los casos que proceda ya como todo hay excepciones para hacer eso de estos procedimientos.

Es significativo señalar que para poder aplicar las MASC en materia, se tiene que seguir ciertas reglas para su operatividad en México, por ejemplo, se necesita conocer que la controversia no acepte derechos de terceros, debe ser a solicitud de las partes respetando el principio de voluntariedad, la cuantía involucrada, delito debe ser perseguible por querella o en determinados casos que la conducta tipificada permita el otorgamiento del perdón a quien cometido la conducta, entre otros requisitos.

Toda persona puede ser uso de un mecanismo alternativo dentro de la Fiscalía General del Estado de Tabasco y de sus Centros de Procuración de Justicia adscrito en todo el Estado, donde existen facilitadores o especialista adscritos al Órgano el cual el primer paso acceder a la atención es realizar una denuncia o querella y solicitarlo desde el inicio de su trámite participar en un mecanismo alternativo.

Al participar en un mecanismo alternativos se rompe con unos de los paradigmas del anterior proceso que nos regia donde se buscaba condenar al agresor y no se contaba con la oportunidad de ser escuchado o el acusado pueda explicar el porqué de su conducta, en ocasiones reconoce su culpa y acepta hacer la reparación del daño el cual no siempre es de tipo económica sino en ocasiones el solicitante del servicio busca su tranquilidad y seguridad.

Una vez suscrito los acuerdos reparatorio se da un seguimiento que en líneas anteriores ya se explicaron, estos lineamientos y herramientas con la que cuenta el órgano y cuando se actúa de manera eficaz y pronta se puede llegar a cumplir todos y cada uno de las responsabilidades y compromisos detallado en el acuerdo y si en su caso esto no se lograr pues informar en debido tiempo el incumplimiento o cumplimiento del mismos al fiscal del ministerio público o al Juez que corresponda, lo cual el caso que sea se actualiza de manera inmediata la base que lleva el Órgano una vez teniendo el conocimiento para que la información de los usuarios en caso de ocuparse existan los registros actualizados. 


\section{DISCUSIÓN}

Con los datos anteriores podemos notar que apartir de la reforma de la reforma del 2008 a la Constitución Política de los Estados Unidos Mexicanos (CPEUM) en su artículo 17, cuarto párrafo, da la creación de la LNMASCMP, las personas considerabas víctimas u ofendidas por la comisión de un delito, pueden acceder a un mecanismo alternativo (mediación, conciliación o junta restaurativa) con el uso de su voluntad, donde el único fin es que su daño sea reparado y tenga solución a su conflicto de una manera pronta y eficaz haciendo uso de esto métodos.

Se espera que los usuarios tengan conocimiento que después de la suscripción de un acuerdo reparatorio diferido, ante la institución de procuración de Justicia del Estado de Tabasco ya sea al inicio de su investigación o Juez competente se le da un seguimiento por la unidad administrativa denominada Órgano Especializado en Mecanismos Alternativos de Solución de Controversias en Materia Penal, el cual cuenta con áreas administrativas y un manual para funcionamiento adecuado, donde se lleva un registro de todos los ingresos de los procedimientos.

Que existen herramientas de apoyo y reuniones de revisión para poder hacer cumplir dichos responsabilidades y obligaciones estampadas, si una vez agotados las herramientas no se logra el cumplimiento su procedimiento en la etapa en que se encuentre sigue su trayectoria en el estado en que se encontraba al acudir al Órgano.

El área del seguimiento de acuerdo reparatorio del Órgano se localiza en oficina central y sus diferentes colaboradores (facilitadores) en cada una de las cabeceras municipales del Estado.

\section{REFERENCIAS}

1. Basulto Arroyo, Johanna Guadalupe. (2019). Los métodos alternativos de justicia y la reforma del sistema penal. México: IAP.

2. Cámara de Diputados. (2014). Ley Nacional de Mecanismos Alternativos de Solución de Controversias en Materia Penal.

3. Cámara de Diputados . (2008). Constitucion Politica de los Estados Unidos Mexicanos.

4. Fiscalía General del Estado de Tabasco. (2019). Boletín Informativo $N^{\circ} 2479$. En lo que va de abril, recupera Fiscalía casi dos millones de pesos en favor de víctimas del delito. México.

5. Fiscalía General del Estado de Tabasco. (2020). Manual de estructura,funciones y procedimientos de la direccion de mecanismos alternativos de solución de controversias en materia penal. México: Manual EFP DMASC.

6. Horvitz Lennon, María Inés \& López Masle Julián. (2003). Derecho procesal penal chileno. Chile: Editorial Jurídica de Chile.

7. Jimenez Martínez, Javier. (2016). Las Formas Alternas para la Solucion de Controversias Penales. Mexico, Flores Editores y Distribuidor.

8. Naciones Unidas. (1985). Declaración sobre los principios fundamentales de justicia para las víctimas de delitos y del abuso de poder.

9. Periódico Oficial del Estado. (2014). Ley Orgánica de la Fiscalía General del Estado de Tabasco.

10. Silva-Hernández, F. (2020). Hacia una comprensión del conflicto en justicia alternativa. Revista de Investigaciones Universidad del Quindio, 32(1), 61-65. https://doi.org/10.33975/riuq.vol32n1.435

11. Vasconcelos Méndez, Ruben. (2012). Principio de oportunidad y salidas alternativas en el proceso penal mexicano. Mexico: Flores. 